\title{
Synthesis of graphene oxide-intercalated $\alpha$-hydroxides by metathesis and their decomposition to graphene/metal oxide composites
}

\author{
C. Nethravathi ${ }^{a}$, Michael Rajamathi ${ }^{a, *}$, N. Ravishankar ${ }^{b}$, Lubna Basit ${ }^{c}$, Claudia Felser ${ }^{c}$ \\ a Materials Research Group, Department of Chemistry, St. Joseph's College, 36 Lalbagh Road, Bangalore 560 027, India \\ ${ }^{\mathrm{b}}$ Materials Research Centre, Indian Institute of Science, Bangalore 560 012, India \\ c Institute for Inorganic and Analytical Chemistry, Johannes Gutenberg University, 55099 Mainz, Germany
}

\section{A R T I C L E I N F O}

Article history:

Received 26 May 2010

Accepted 26 July 2010

Available online 2 August 2010

\begin{abstract}
A B S T R A C T
Graphene oxide-intercalated $\alpha$-metal hydroxides were prepared using layers from the delaminated colloidal dispersions of cetyltrimethylammonium-intercalated graphene oxide and dodecylsulfate-intercalated $\alpha$-hydroxide of nickel/cobalt as precursors. The reaction of the two dispersions leads to de-intercalation of the interlayer ions from both the layered solids and the intercalation of the negatively charged graphene oxide sheets between the positively charged layers of the $\alpha$-hydroxide. Thermal decomposition of the intercalated solids yields graphene/nanocrystalline metal oxide composites. Electron microscopy analysis of the composites indicates that the nanoparticles are intercalated between graphene layers.
\end{abstract}

(c) 2010 Elsevier Ltd. All rights reserved.

\section{Introduction}

Layered composites in which the layers from two different layered solids are stacked alternatively are important as they would exhibit unique physiochemical properties such as 2-D magnetism and conductivity due to the combined effect of the properties of the structural unit layers. Metastable mixed layered composites have been prepared through soft chemical routes. Layered composites, such as clay/metal hydroxide [1-3], $\mathrm{MoS}_{2} / \mathrm{Ni}(\mathrm{OH})_{2}$ or $\mathrm{Co}(\mathrm{OH})_{2}$ [4], graphite oxide/birnessite [5], have been prepared by intercalation of $\mathrm{M}^{2+}$ ions in the interlayer of the host followed by titrating the product with alkali. Birnessite/ $\mathrm{NiOH}_{2}$ [6], birnessite/ $\mathrm{LiAl}_{2}(\mathrm{OH})_{6}$ [7], clay/ $\mathrm{LiAl}_{2}(\mathrm{OH})_{6}$ [8] and clay/SnO ${ }_{2}$ [9] have been prepared by intercalation followed by hydrothermal treatment. Graphite/ $\mathrm{NiOH}_{2}$ has been prepared through intercalation of $\mathrm{Ni}^{2+}$ ions in graphite and subjecting the product to electrochemical cycling in the presence of alkali [10]. The synthesis approaches to such composites are restricted to a few select systems and are not general in nature.

Delamination of layered solids yields solvated monolayers that could be used as synthons in the preparation of layered composites. We have shown that interstratified composites of similarly charged layers of layered solids having related structures [11-13] and of layered solids having different structures [14] can be prepared starting from the monolayer colloidal dispersions of layered solids. We reported the preparation of 1-D solid solutions of $\alpha$-hydroxides of $\mathrm{Ni}$ and Co [11], two different layered double hydroxides (LDHs), namely $\mathrm{Mg}-\mathrm{Al}$ and Co-Al LDHs [12], two different cationic clays - a dioctahedral clay and a trioctahedral clay [13] and graphite oxide (GO)/ smectite [14] through the delamination-costacking approach.

It is also possible to make layer-by-layer composites of two oppositely charged layered solids through delamination $[15,16]$. We have recently shown that positively charged GO layers could be introduced into the interlayer of exfoliated an-

* Corresponding author: Tel.: +91 80 22211429; fax: +91 8022245831.

E-mail address: mikerajamathi@rediffmail.com (M. Rajamathi). 0008-6223/\$ - see front matter (c) 2010 Elsevier Ltd. All rights reserved. doi:10.1016/j.carbon.2010.07.047 
ionic clay by ion-exchange [17]. In this work, we describe a metathesis route to layer-by-layer composites. If we denote the anionic and cationic layered solids as $\mathrm{L}^{+}-\mathrm{A}^{-}$and $\mathrm{L}^{-}-\mathrm{C}^{+}$ where $\mathrm{L}^{+}$and $\mathrm{L}^{-}$are the layers of the anionic and cationic layered solids and $\mathrm{A}^{-}$and $\mathrm{C}^{+}$are the interlayer anions and cations then we can visualize a reaction of the type

$\mathrm{L}^{+}-\mathrm{A}^{-}+\mathrm{L}^{-}-\mathrm{C}^{+} \rightarrow \mathrm{L}^{+}-\mathrm{L}^{-}+\mathrm{AC}$

if $\mathrm{A}^{-}$and $\mathrm{C}^{+}$are surfactant ions then the reaction may be carried out in an organic solvent in which both the layered solids would delaminate. The product AC being highly soluble in the organic solvent, the reaction will be driven forward to yield $\mathrm{L}^{+}-\mathrm{L}^{-}$, the layer-by-layer composite.

Graphene (G) based composites are important as they are potent materials for applications in varied fields such as nanoelectronics, sensors, batteries, supercapacitors, hydrogen storage, catalysts, catalyst supports, adsorbents and magnetic materials [18]. Graphite/polymer [19], graphite/ nanoparticle composites [20], supercapacitor and battery electrode materials [21,22] have been fabricated starting from GO as it is dispersible in water and alkaline medium [23] and in organic solvents after modification with amines [24-26].

$\alpha$-Hydroxides of nickel and cobalt are important electrode materials in alkaline secondary batteries $[27,28]$. In addition, these solids decompose at relatively low temperatures $\left(<300^{\circ} \mathrm{C}\right)$ to give the corresponding oxides. The hydroxides of nickel and cobalt crystallize in different polymorphic modifications, mainly the $\beta$ - and the $\alpha$-form $[29,30]$. The $\beta$-form is of the formula $\mathrm{M}(\mathrm{OH})_{2}$ and it is an ordered stacking of neutral layers of the composition $\left[\mathrm{M}(\mathrm{OH})_{2}\right]$ with an interlayer spacing of $4.6 \AA$. The $\alpha$-form is a hydroxyl-deficient compound and consists of a stacking of positively charged layers of composition $\left[\mathrm{M}(\mathrm{OH})_{2-\mathrm{x}}\left(\mathrm{H}_{2} \mathrm{O}\right)_{\mathrm{x}}\right]^{\mathrm{x}+}$, which intercalate anions such as $\mathrm{NO}_{3}^{-}, \mathrm{Cl}^{-}, \mathrm{OAc}^{-}, \mathrm{SO}_{4}^{2-}$, etc. along with water molecules in the interlayer region to restore charge neutrality. Consequently, the $\alpha$-hydroxides have a higher interlayer spacing, which varies with the size of the interlayer anion. On intercalation of surfactant anions, dodecylsulfate (DS) and dodecylbenzene sulfonate (DBS), these solids delaminate to give a colloidal dispersion of layers in organic solvents such as 1-butanol [31]. It would be interesting to prepare layer-by-layer composites of $\alpha$-hydroxides and GO. Thermal decomposition of these composites would reduce GO to $G$ and convert hydroxides to oxides resulting in G-metal oxide composites. In this paper, we report the metathesis between alkylammonium ion-intercalated GO and DS-intercalated $\alpha$-hydroxide of nickel/cobalt that yields $\mathrm{GO} / \alpha$-hydroxide layer-by-layer composites (GO sheets intercalated $\alpha$-hydroxides). These composites on heating in different atmospheres yield interesting G/inorganic material composites.

\section{Experimental section}

\subsection{Synthesis of the precursors}

DS-intercalated $\alpha$-divalent metal hydroxides were prepared by the addition of $35 \mathrm{ml}$ of a solution containing metal acetate, $\mathrm{M}(\mathrm{OAc})_{2} \cdot 4 \mathrm{H}_{2} \mathrm{O}[\mathrm{M}=\mathrm{Ni}, \mathrm{Co}]$ and the surfactant, NaDS in the mole ratio 1:0.9 into $50 \mathrm{ml}$ of $0.5 \mathrm{M} \mathrm{NH}_{3}$ solution with constant stirring [31]. The solid product formed was immediately centrifuged, washed free of anions with water followed by acetone and dried in air at room temperature. Hereafter these surfactant-intercalated $\alpha$-hydroxides are referred to as $\mathrm{Ni}$ $\mathrm{OH}-\mathrm{DS}$ and $\mathrm{Co}-\mathrm{OH}-\mathrm{DS}$.

The method due to Hummers and Offeman [32] was adopted to prepare GO from graphite powder $(10-20 \mu \mathrm{m})$ supplied by Graphite India Limited, Bangalore, India. One gram of graphite powder was added to $23 \mathrm{ml}$ of cooled $\left(0^{\circ} \mathrm{C}\right)$ concentrated $\mathrm{H}_{2} \mathrm{SO}_{4}$. Three grams of $\mathrm{KMnO}_{4}$ was added gradually with stirring and cooling, so that the temperature of the mixture was maintained below $20^{\circ} \mathrm{C}$. The mixture was then stirred at $35^{\circ} \mathrm{C}$ for $30 \mathrm{~min}$. Forty-six milliliters of distilled water was slowly added to cause an increase in temperature to $98^{\circ} \mathrm{C}$ and the mixture was maintained at that temperature for $15 \mathrm{~min}$. The reaction was terminated by adding $140 \mathrm{ml}$ of distilled water followed by $10 \mathrm{ml}$ of $30 \% \mathrm{H}_{2} \mathrm{O}_{2}$ solution. The solid product was separated by centrifugation, washed repeatedly with $5 \% \mathrm{HCl}$ solution until sulfate could not be detected with $\mathrm{BaCl}_{2}$, then washed 3-4 times with acetone and dried in an air oven at $65^{\circ} \mathrm{C}$ overnight.

Cetyltrimethylammonium (CTA)-intercalated GO was prepared by stirring GO in an aqueous solution of cetyltrimethyl ammonium bromide ( 5 times the ion-exchange capacity of GO) for 3 days $[25,26,33]$. The solid thus obtained was washed with water till the $\mathrm{pH}$ of the washings turned $\sim 7$ and finally with acetone and dried at $65^{\circ} \mathrm{C}$. This sample is hereafter referred to as GO-CTA.

\subsection{Delamination and colloidal dispersion}

The delamination and monolayer colloidal dispersion of GOCTA were achieved by subjecting a mixture of $100 \mathrm{mg}$ of the sample and $100 \mathrm{ml}$ of the solvent, 1-butanol, to sonication ( $35 \mathrm{kHz}$ ) at room temperature for $30 \mathrm{~min}$. Monolayer colloidal dispersion of Ni-OH-DS/Co-OH-DS was obtained by subjecting a mixture of $133 / 125 \mathrm{mg}$ of the sample and $400 \mathrm{ml}$ of 1 butanol to sonication $(35 \mathrm{kHz})$ at room temperature for $30 \mathrm{~min}$. Composite formation requires that the number of moles of surfactants $\left(\mathrm{CTA}^{+}\right.$and $\left.\mathrm{DS}^{-}\right)$is equal. From the charge compensation calculations, it was estimated that $100 \mathrm{mg}$ of GO-CTA is required to compensate the charges on $133 \mathrm{mg}$ of $\mathrm{Ni}-\mathrm{OH}-\mathrm{DS} / 125 \mathrm{mg}$ of Co-OH-DS.

\subsection{Metathesis}

The colloidal dispersions of GO-CTA and M-OH-DS $(\mathrm{M}=\mathrm{Ni}$ or Co) were mixed and sonicated for $30 \mathrm{~min}$. The resulting dispersion was then stirred at $70{ }^{\circ} \mathrm{C}$ for 3 days. The black precipitate (GO-intercalated $\alpha$-hydroxide) obtained at the end of 3 days was repeatedly washed with acetone to remove the surfactants and the solid was dried in air at room temperature.

\subsection{Thermal decomposition of the GO-intercalated $\alpha$ - hydroxides}

GO-intercalated $\alpha$-hydroxides were subjected to isothermal heating in air/nitrogen atmosphere. The samples were held at $300^{\circ} \mathrm{C}$ for $2 \mathrm{~h}$. 


\subsection{Characterization}

The powder X-ray diffraction (pXRD) patterns of the samples were recorded using a PANalytical X'pert Pro diffractometer ( $\mathrm{Cu} \mathrm{K} \alpha$ radiation, secondary graphite monochromator, $2^{\circ} 2 \theta$ / min). The infrared (IR) spectra of the samples were recorded on a Nicolet Impact 400D FTIR spectrometer ( $\mathrm{KBr}$ pellets, $4 \mathrm{~cm}^{-1}$ resolution). The samples were also characterized by transmission electron microscopy (TEM) using a JEOL 200CX microscope operated at $160 \mathrm{kV}$. Superconducting quantum interference device (SQUID) magnetometry was carried out using a Quantum Design MPMS-XL-5 at 300 and $5 \mathrm{~K}$.

The different process involved in the metathetic reaction between the colloidal dispersions of the surfactant-intercalated GO and metal hydroxide and the decomposition of GO-intercalated $\alpha$-hydroxide to $\mathrm{G} /$ metal oxide composites is schematically shown in Fig. 1.

\section{Results and discussion}

Fig. $2 \mathrm{a}, \mathrm{b}$ and $\mathrm{c}$ are the pXRD patterns of GO-CTA, Co-OH-DS and Ni-OH-DS with basal spacings of 30,26 and $31 \AA$, respectively. While GO-CTA (Fig. 2a) shows only the 001 reflections, the $\alpha$-hydroxides (Fig. $2 b$ and c) show, in addition to the 001 reflections, the characteristic saw-tooth-shaped broad peaks due to the 2-D (10) and (11) reflections arising out of turbostratic disorder [34,35].

The pXRD patterns of the GO-intercalated $\alpha$-hydroxides obtained by metathesis are shown in Fig. 3. In order to understand the mechanism of formation of the composite, intermediates collected at different intervals were studied. The absence of basal reflections and the presence of the sawtooth-shaped broad reflections (Fig. 3a) starting at $\sim 33^{\circ}$ and $\sim 59^{\circ}$ in the case of the sample obtained after $24 \mathrm{~h}$ of reaction suggests that the reactants still exist in delaminated state. At the end of $48 \mathrm{~h}$ (Fig. $3 \mathrm{~b}$ ) the layers have reacted to form the composite with a basal spacing of $13.5 \AA$ that corresponds to the sum of layer thicknesses of the $\alpha$-hydroxide (4.6 $\AA$ ) and GO ( 6-7 $\AA$ ). The reduced basal spacing of the composite compared to that of the precursors (Fig. 2) indicate that the surfactant ions are no longer present in the interlayer region of the layered solids. Possibly, negatively charged GO sheets are present in the interlayer region of the $\alpha$-hydroxides compensating for the positive charge on the anionic clay layers. The absence of GO-related reflections in the composite further indicates this possibility. As the reaction is carried on fur-

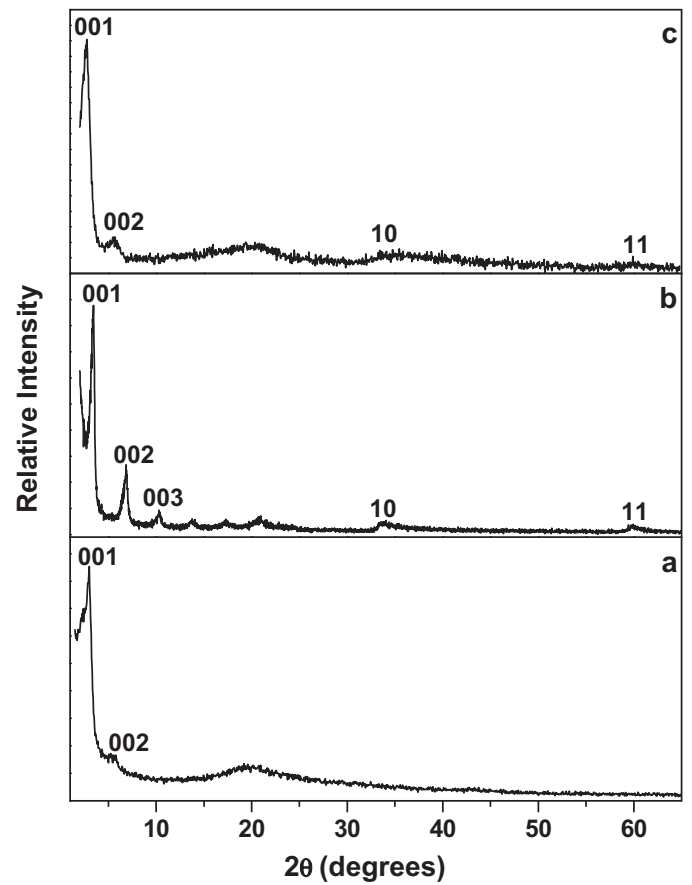

Fig. 2 - pXRD patterns of the as-prepared GO-CTA (a) Co-OHDS (b) and Ni-OH-DS (c).

ther, the basal spacings of GO-intercalated nickel hydroxide (Fig. 3c) and GO-intercalated cobalt hydroxide (Fig. 3d) decrease to 8.2 and $7.9 \AA$, respectively. The saw-tooth-shaped broad reflections starting at $\sim 33^{\circ}$ and $\sim 59^{\circ}$ in both the samples indicate that the layer structure of the anionic clay is intact. The reduced basal spacing in the composites obtained after 3 days is due to the partial reduction of the intercalated GO layers. In order to ascertain the nature of the GO layers present in the intercalated solid, we leached out the inorganic matter from the solid in acid. The pXRD pattern of the sample after acid leaching (Fig. 3e) matches that of partially reduced GO with a basal spacing of $4.3 \AA$. As the metathetic reaction was carried in butanol, a reducing solvent, the basal functional groups of the GO layers have been partially reduced. Similar reductions have been reported earlier in other reducing media [36,37]. Thus the GO layers in the intercalated solids have a smaller layer thickness compared to the pristine GO. This explains the low observed basal spacing for the GO-intercalated $\alpha$-hydroxides at the end of 3 days. The carboxylic acid groups at the edges of the GO layers are not

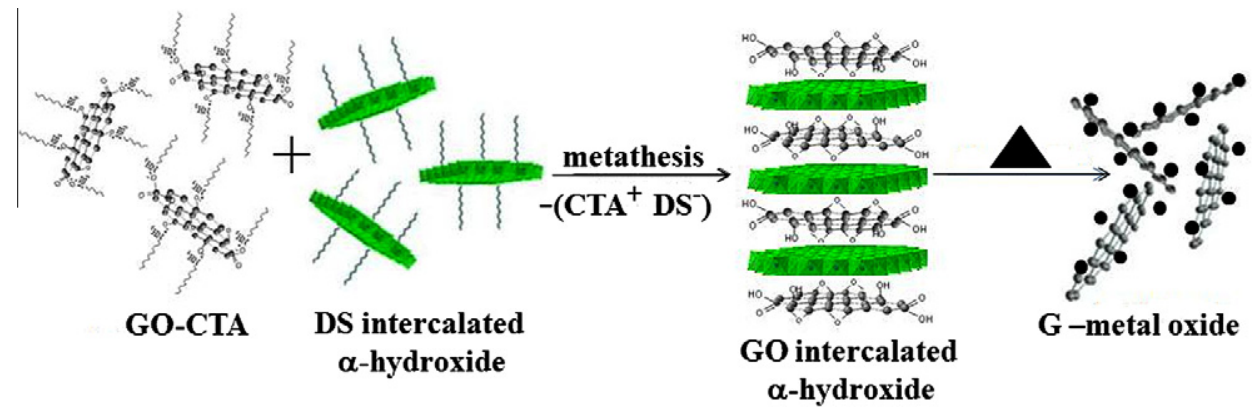

Fig. 1 - Schematic representation of the process involved in the formation of GO-intercalated $\alpha$-hydroxide. 


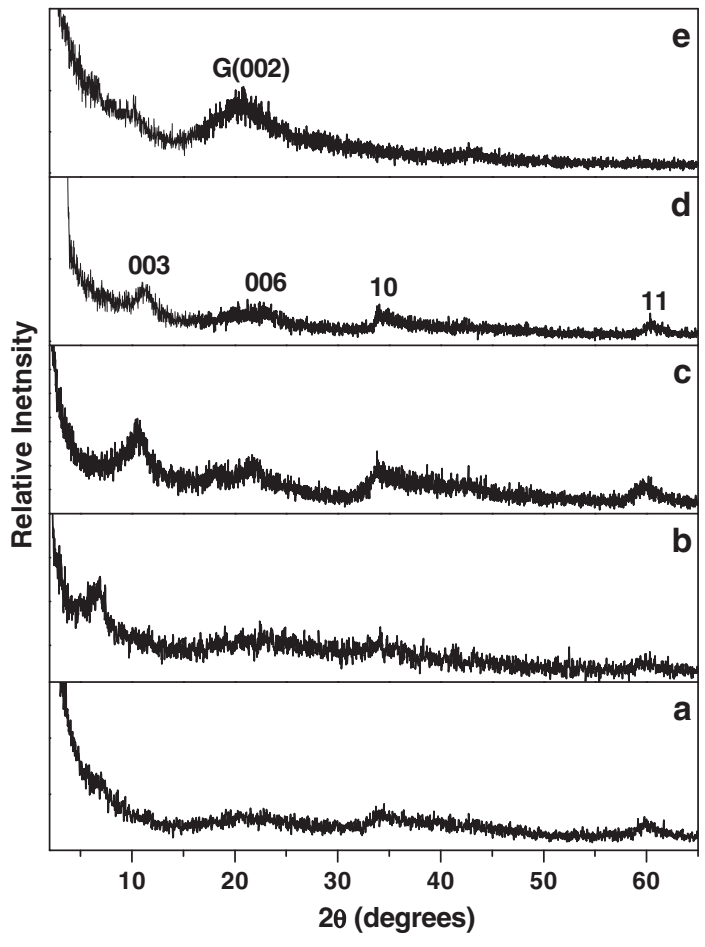

Fig. 3 - pXRD patterns of the GO-CTA and Ni-OH-DS reacted for 1 (a) and 2 (b) days; Go-intercalated $\alpha$-nickel hydroxide (c) and GO-intercalated $\alpha$-cobalt hydroxide (d) obtained by metathesis at the end of 3 days; GO obtained after acid leaching of GO-intercalated $\alpha$-cobalt hydroxide (e).

expected to get reduced under these conditions and these groups contribute to the negative charge on the GO layers which counters the positive charge on the hydroxide layers. The layer charge (the exchangeable protons) on the partially reduced GO, obtained after leaching out the hydroxide layers, estimated by potentiometric titration using $\mathrm{NaOH}$, [33-38] was $3.9 \mathrm{mmol} \mathrm{g}^{-1}$. As the GO-intercalated $\alpha$-hydroxide has $39 \%$ by mass of GO the total negative charge from the GO layers per gram of the composite is $1.6 \mathrm{mmol}$. The total positive charge from the $\alpha$-hydroxide layers per gram of the composite is $\sim 2 \mathrm{mmol}$. Thus $\sim 80 \%$ of the positive charges on the hydroxide layers are compensated by the carboxyl groups of the reduced GO layers.

In order to see if reduction of GO layers occurs under the reaction conditions we heated GO-CTA in 1-butanol at $70^{\circ} \mathrm{C}$ for $72 \mathrm{~h}$. The pXRD pattern and the IR spectrum of the product obtained are shown in Fig. 4 . The peak at $\sim 4 \AA$ in the pXRD pattern (Fig. 4a) due to partially reduced GO confirms that reduction of GO occurs at the conditions of the composite formation reaction. There is a small amount of unreduced GO as indicated by the peak at $7.7 \AA$ A. In the IR spectrum (Fig. 4b) we observe weak peaks corresponding to carboxylic acid groups suggesting that these groups are not reduced under the conditions of composite formation.

In Fig. 5 the IR spectrum of GO-intercalated $\alpha$-nickel hydroxide is compared with the spectra of the precursors GO-CTA and Ni-OH-DS. In the case of GO-CTA (Fig. 5a), we observe a strong and broad absorption at $3500 \mathrm{~cm}^{-1}$ due to $\mathrm{H}$ bonded $\mathrm{O}-\mathrm{H}$ and $\mathrm{N}-\mathrm{H}$ stretching vibrations. The $\mathrm{O}-\mathrm{H} / \mathrm{N}-\mathrm{H}$

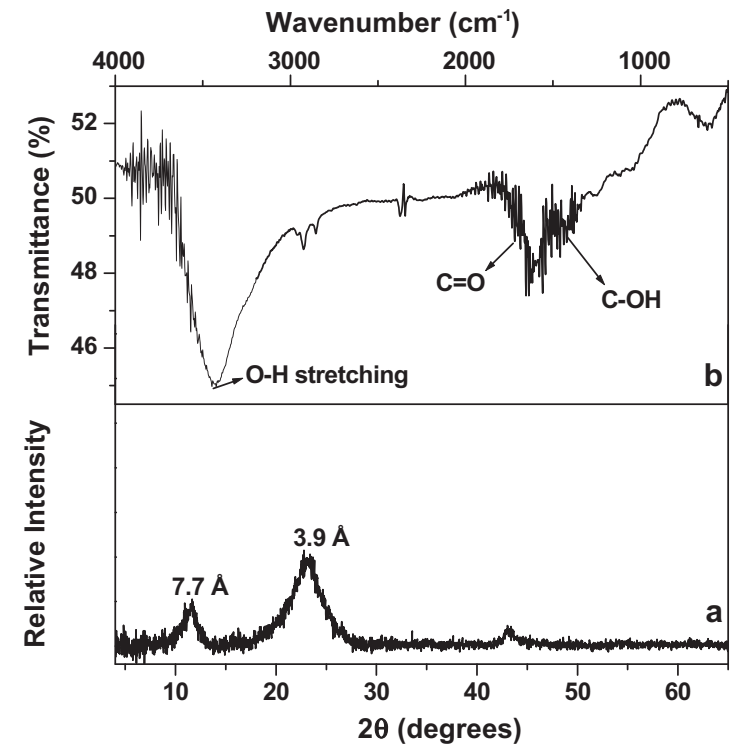

Fig. 4 - pXRD pattern (a) and IR spectrum (b) of the sample obtained on heating GO-CTA at $70^{\circ} \mathrm{C}$ for $72 \mathrm{~h}$.

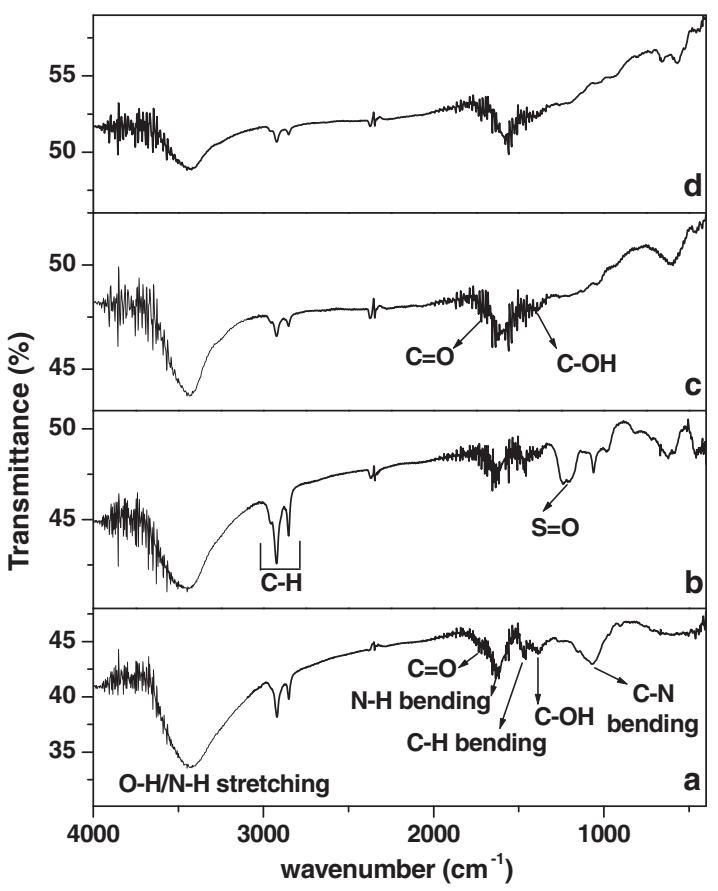

Fig. 5 - IR spectra of GO-CTA (a), Ni-OH-DS (b), GOintercalated $\alpha$-nickel hydroxide (c) and Go-intercalated $\alpha$ cobalt hydroxide (d).

bending and $\mathrm{C}-\mathrm{N}$ bending vibrations are observed at 1600 and $1050 \mathrm{~cm}^{-1}$ respectively. In addition, the absorptions specific to $\mathrm{GO}$ - at $1700 \mathrm{~cm}^{-1}$ due to $\mathrm{C}=\mathrm{O}$ stretching and at $1376 \mathrm{~cm}^{-1}$ due to $\mathrm{C}-\mathrm{OH}$ bending - are also observed. In addition to the absorption at $1600 \mathrm{~cm}^{-1}$, the alkylammonium ion is expected to give a weak absorption in the range of 1550$1480 \mathrm{~cm}^{-1}$. We do not observe this absorption because either it is too weak or it overlaps with the $\mathrm{C}-\mathrm{H}$ bending absorption peak centered at $1470 \mathrm{~cm}^{-1}$. The $\mathrm{C}-\mathrm{H}$ stretching vibrations of 
the alkyl chain of the amine are observed at around 2850$2900 \mathrm{~cm}^{-1}$. The presence of the absorptions due to $\mathrm{N}-\mathrm{H}$ and $\mathrm{C}-\mathrm{H}$ bonds is indicative of intercalation of CTA ions in the interlayer of GO.

In the case of Ni-OH-DS (Fig. 5b) we observe a strong and broad absorption at $3500 \mathrm{~cm}^{-1}$ due to hydrogen bonded hydroxyl groups, and the $\mathrm{S}=\mathrm{O}$ stretching vibration of the sulfate group at $1200 \mathrm{~cm}^{-1}$. In both GO-CTA and Ni-OH-DS the C-H stretching vibrations of the methylene groups of the alkyl chains of the surfactant are observed at $2850-2950 \mathrm{~cm}^{-1}$. The nature of the $\mathrm{C}-\mathrm{H}$ stretching absorption band (strong peaks at $2850-2950 \mathrm{~cm}^{-1}$ ) suggests that the alkyl chains take up all trans conformation $[39,40]$. If the chains were disordered, the peak at $2920 \mathrm{~cm}^{-1}$ would be broad and weak.

The IR spectrum of GO-intercalated $\alpha$-nickel hydroxide (Fig. 5c) shows no features related to the surfactant anions $\left(\mathrm{CTA}^{+}\right.$and $\left.\mathrm{DS}^{-}\right)$indicating the removal of surfactant ions. The bands due to $\mathrm{C}=\mathrm{O}$ stretching and $\mathrm{C}-\mathrm{OH}$ bending of the GO layers are observed at 1700 and $1376 \mathrm{~cm}^{-1}$ indicating the presence of GO layers in the sample. There are no absorptions corresponding to any anion such as carbonate. These observations suggest that the negatively charged GO sheets are intercalated in the interlayer region of the hydroxides for charge compensation. The IR spectrum of GO-intercalated $\alpha$-cobalt hydroxide (Fig. $5 \mathrm{~d}$ ) is similar to that of the nickel hydroxide analog.

The bright-field TEM image of the as-prepared GO-intercalated $\alpha$-nickel hydroxide shown in Fig. 6 , indicates the wrinkled sheet structure of the composite extending several microns across.

Heat treatment of the GO-intercalated $\alpha$-hydroxides is expected to reduce GO component of the solid to graphite [41] and to convert the layered hydroxide component to nanoparticles of oxides of cobalt/nickel to give G/metal oxide inorganic composites. With the objective of making these Gbased composites, the GO-intercalated $\alpha$-hydroxides were

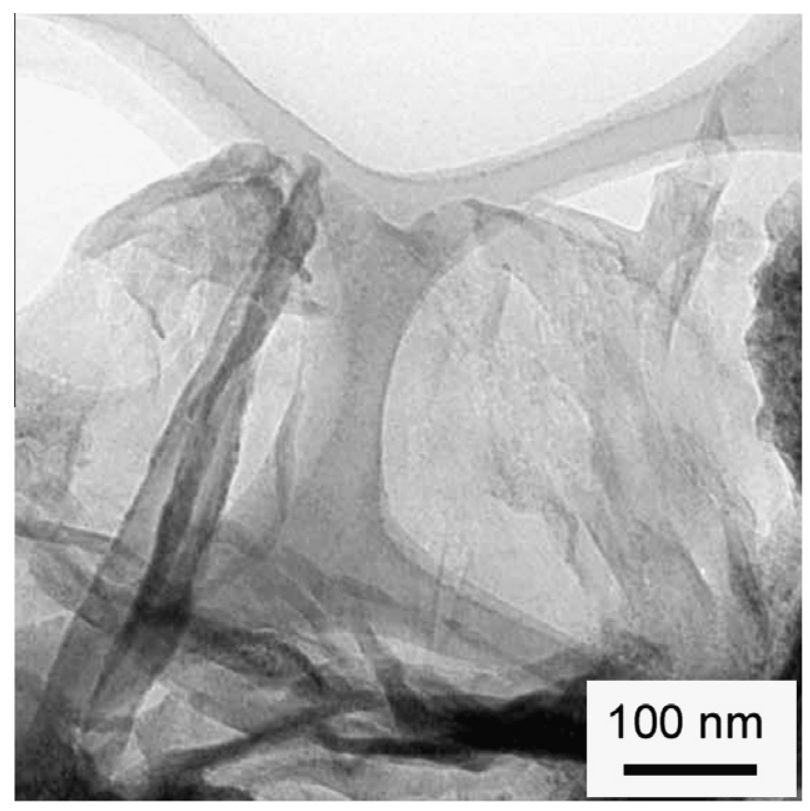

Fig. 6 - Bright-field TEM image of the as-prepared GOintercalated $\alpha$-nickel hydroxide. subjected to decomposition at $300^{\circ} \mathrm{C}$ in different atmospheres. The pXRD patterns of the heat-treated samples are shown in Fig. 7. On decomposition in air, the GO-intercalated

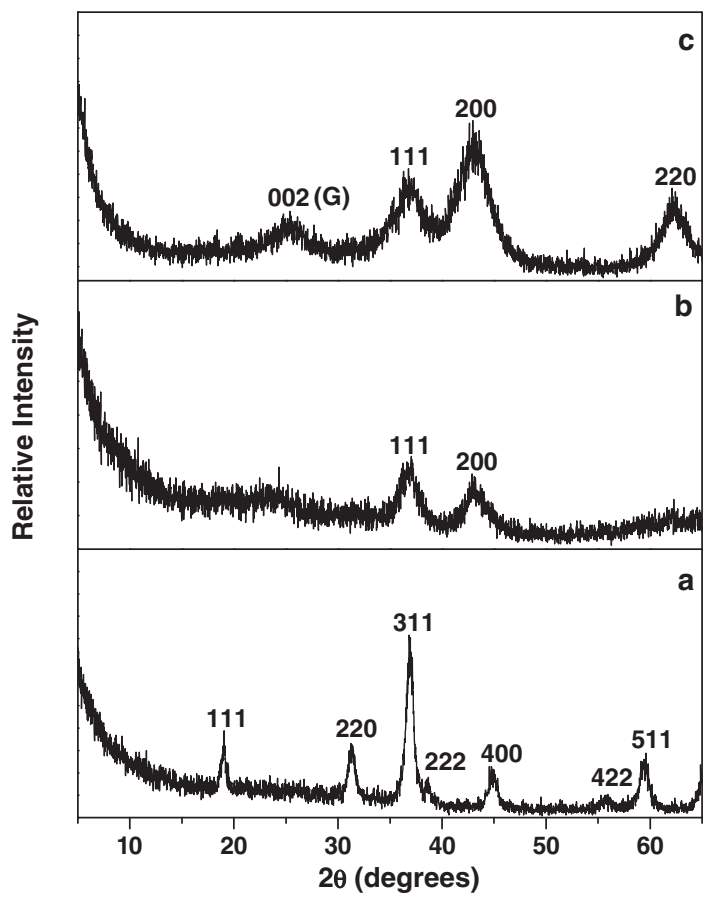

Fig. 7 - pXRD patterns of the GO-intercalated $\alpha$-cobalt hydroxide heated at $300{ }^{\circ} \mathrm{C}$ in air (a) and in nitrogen (b) and Go-intercalated $\alpha$-nickel hydroxide heated at $300{ }^{\circ} \mathrm{C}$ in air (c).

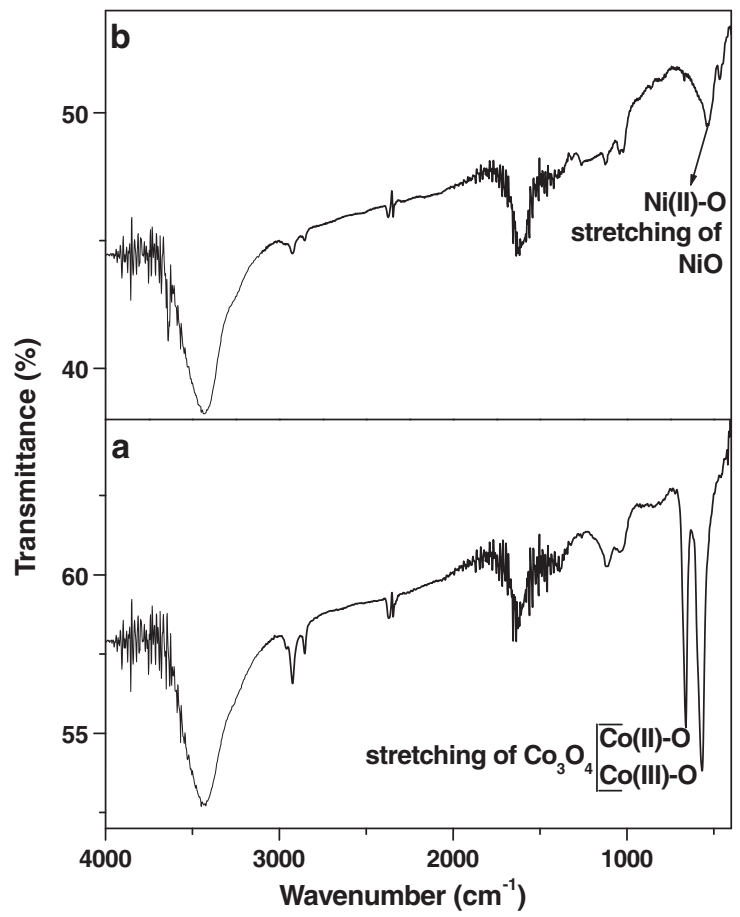

Fig. 8 - IR spectra of GO-intercalated $\alpha$-cobalt hydroxide (a) and GO-intercalated $\alpha$-nickel hydroxide (b) heated at $300{ }^{\circ} \mathrm{C}$ in air. 

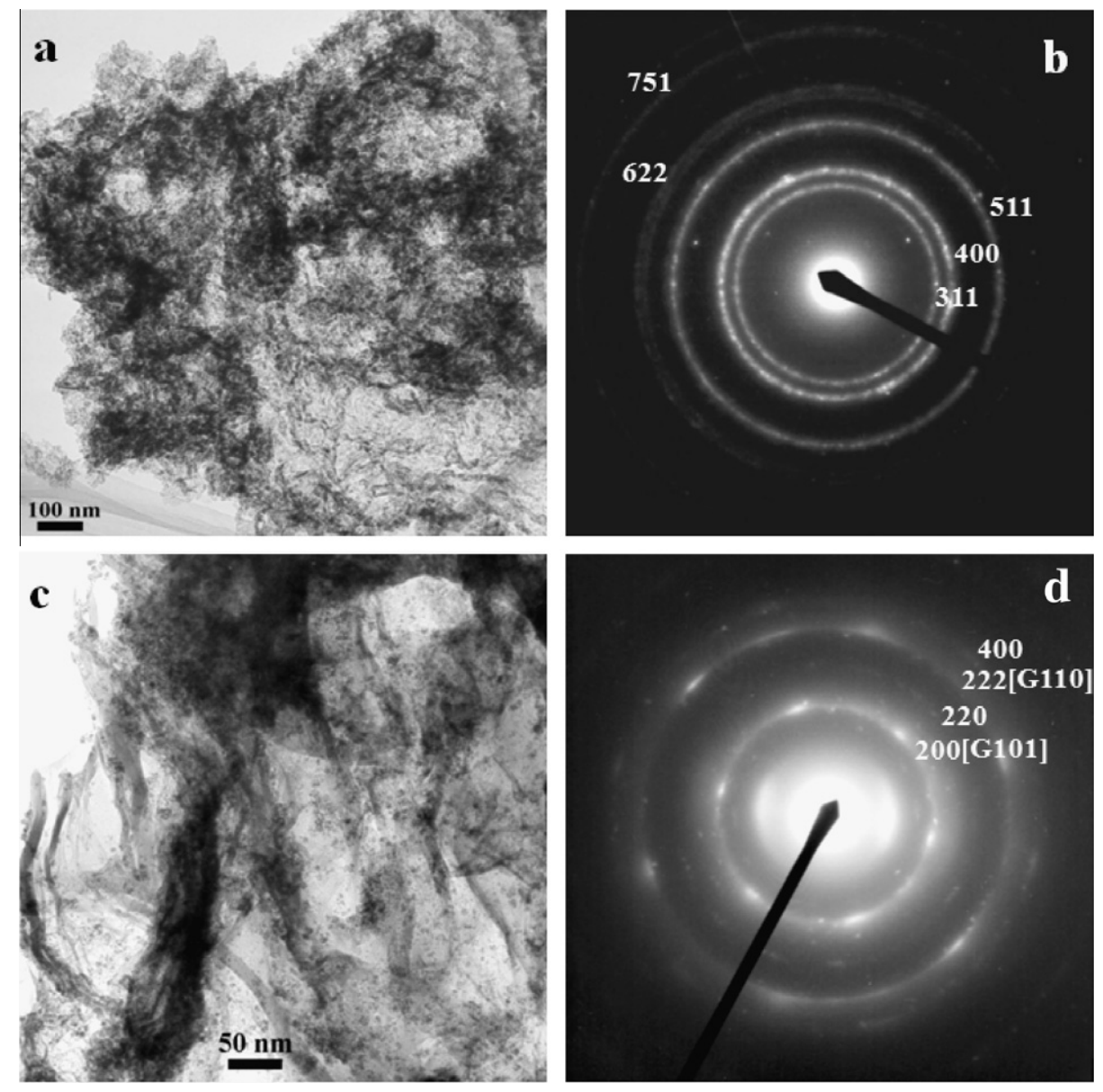

Fig. 9 - Bright-field images of the GO-intercalated $\alpha$-cobalt hydroxide decomposed at $300{ }^{\circ} \mathrm{C}$ in air (a) and the corresponding SAED pattern obtained from the particles (b). The image from the sample heated in nitrogen atmosphere (c) and the corresponding SAED pattern obtained from the particles (d).

$\alpha$-cobalt hydroxide shows peaks due to $\mathrm{Co}_{3} \mathrm{O}_{4}$ (Fig. 7a). No peak due to $\mathrm{GO}$ or graphite is observed indicating that the graphite formed is exfoliated.

When the composite was heated in nitrogen, peaks due to CoO were observed (Fig. 7b). Similarly when GO-intercalated $\alpha$-nickel hydroxide was heated peaks due to NiO were observed (Fig. 7c). In the above two cases a broad peak due to the 002 reflection of graphite was observed at $2 \theta=\sim 25^{\circ}$ ( $d=3.6 \AA$ ). The observed basal spacing is higher than that of well-ordered graphite, $3.35 \AA$. The higher basal spacing may be due to the presence of residual oxygen and hydrogen, indicating incomplete reduction of GO. The 002 reflection of graphite in these samples is very broad, suggesting that the graphite component is very poorly ordered along the stacking direction. This is an indication that these samples comprise exfoliated graphite sheets.

The GO-intercalated $\alpha$-cobalt hydroxide on heating at $300{ }^{\circ} \mathrm{C}$ in air (Fig. 8a) shows strong absorptions at 566 and $662 \mathrm{~cm}^{-1}$ due to decomposition of the layered hydroxide to $\mathrm{Co}_{3} \mathrm{O}_{4}$. The IR spectrum of the decomposition product of GO-intercalated $\alpha$-nickel hydroxide (Fig. $8 b$ ) shows a peak at $532 \mathrm{~cm}^{-1}$ due to Ni-O stretching of NiO. The GO related peaks at 1700 and $1376 \mathrm{~cm}^{-1}$ are absent in both the cases suggesting the reduction of GO to $\mathrm{G}$. The bright field images of the ther- mal decomposition products of GO-intercalated $\alpha$-cobalt hydroxide are shown in Fig. 9a and c. In both the cases we observe crumpled sheets of graphene. The product obtained by heating GO-intercalated $\alpha$-cobalt hydroxide in air (Fig. 9a) shows $\mathrm{Co}_{3} \mathrm{O}_{4}$ particles of diameter $\sim 2 \mathrm{~nm}$ uniformly distributed in the $\mathrm{G}$ matrix. The diffuse rings of the selected area diffraction (SAED) pattern obtained from a particle (Fig. 9b) could be indexed to the cubic spinel phase (JCPDS PDF: 9-418). The sample obtained on heating the composite in nitrogen (Fig. 9c) shows $\mathrm{CoO}$ particles of average diameter $\sim 2 \mathrm{~nm}$ in $\mathrm{G}$ matrix. The hexagonal array of spots in the SAED pattern (Fig. 9d) indicates the presence of ordered graphitic sheets while the faint rings from the cubic CoO phase (JCPDS PDF: 9-402) are also seen in the pattern.

The bright field image of the thermal decomposition product of GO-intercalated $\alpha$-nickel hydroxide in Fig. 10a shows $\sim 2 \mathrm{~nm}$ NiO particles uniformly distributed in exfoliated G matrix. The SAED pattern (Fig. 10b) could be indexed to the cubic NiO phase (JCPDS PDF: 4-0835).

The magnetic properties of the cobalt oxide based composites were investigated by temperature dependent magnetometry. Fig. 11 displays the magnetization curves of $\mathrm{G} /$ $\mathrm{Co}_{3} \mathrm{O}_{4}$ obtained by heating $\mathrm{GO}$-intercalated $\alpha$-cobalt hydroxide in air (Fig. 11a) and G/CoO obtained by heating the same in 

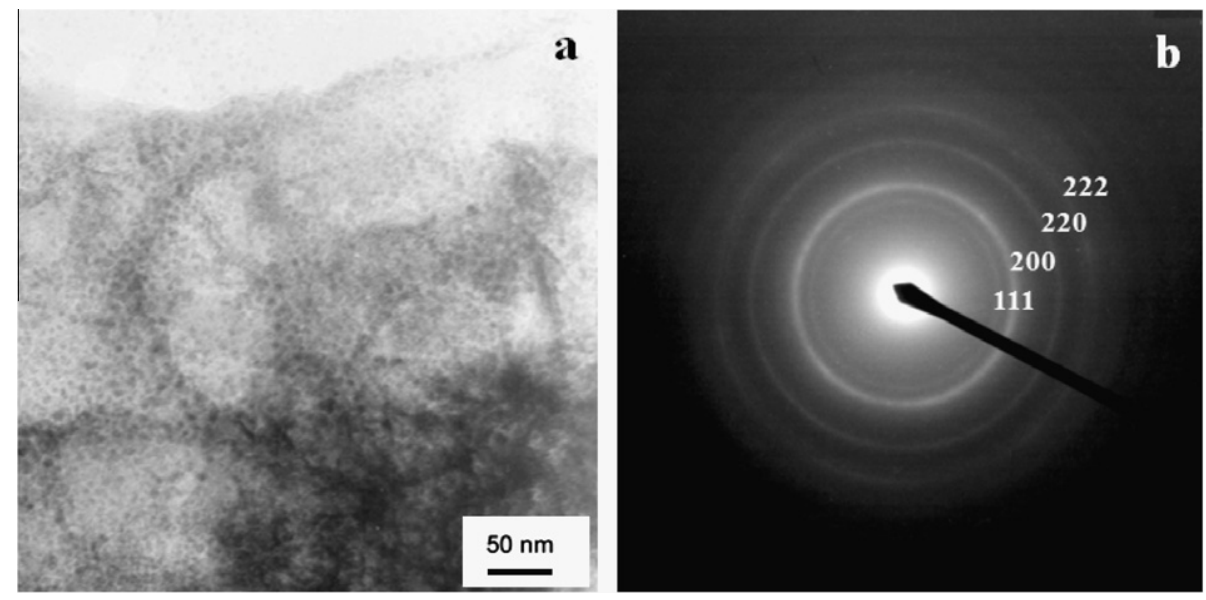

Fig. 10 - Bright-field images of the GO-intercalated $\alpha$-nickel hydroxide decomposed at $300{ }^{\circ} \mathrm{C}$ in air (a) and the corresponding SAED pattern obtained from the particles (b).

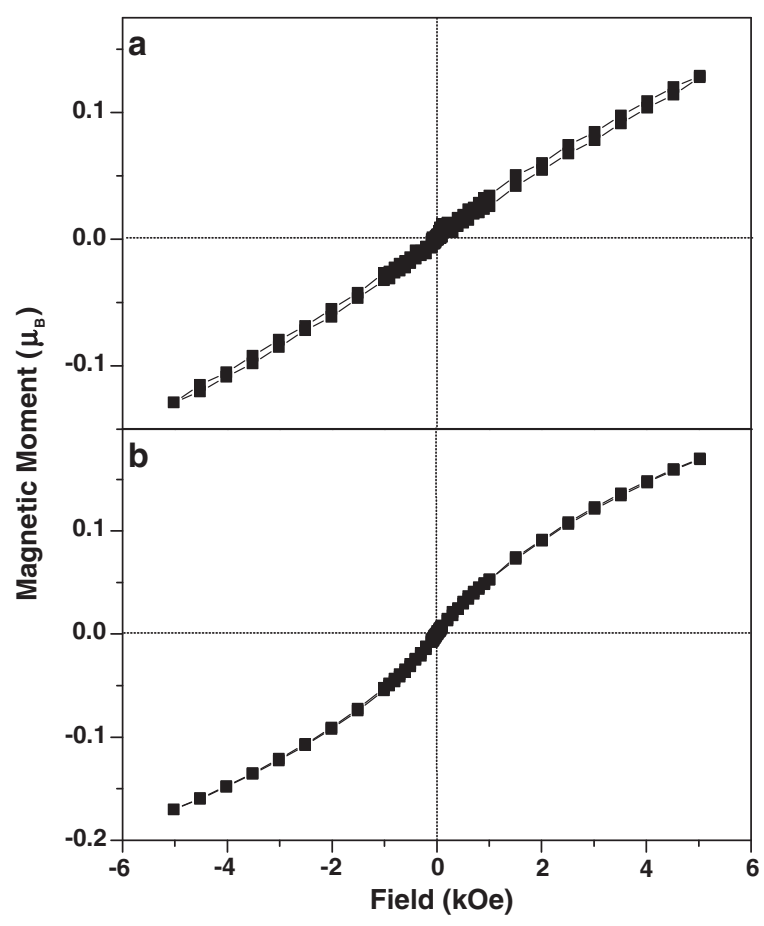

Fig. 11 - Hystersis curves of $\mathrm{G} / \mathrm{Co}_{3} \mathrm{O}_{4}(\mathrm{a})$ and $\mathrm{G} / \mathrm{CoO}$ (b) composites at $5 \mathrm{~K}$.

nitrogen (Fig. 11b) measured at low temperature $(\mathrm{T}=5 \mathrm{~K})$. The $\mathrm{G} / \mathrm{Co}_{3} \mathrm{O}_{4}$ composite exhibits very weak ferromagnetism. It has been earlier observed that nanoparticulate $\mathrm{Co}_{3} \mathrm{O}_{4}$ exhibits weak ferromagnetism due to partial inversion of the spinel structure and/or canted surface spins [42]. The G/CoO composite exhibits superparamagnetism as expected for nanoparticulate $\mathrm{CoO}$.

\section{Conclusions}

Solution based metathesis between macromolecular sheets of CTA-intercalated GO and DS-intercalated $\boldsymbol{\alpha}$-hydroxide could be carried out in an organic solvent. The products were GO sheet-intercalated $\alpha$-hydroxides. These on decomposition yield graphene/metal oxide composites. The metal oxide particles in these composites $-\mathrm{Co}_{3} \mathrm{O}_{4}, \mathrm{CoO}$ and $\mathrm{NiO}$ - are very small $(\sim 2 \mathrm{~nm})$ and these are uniformly distributed in the graphene matrix.

\section{Acknowledgements}

This work was funded by DST, New Delhi. C.N. thanks CSIR, New Delhi for the award of Senior Research Fellowship. C.N. and M.R. thank UGC, New Delhi for having provided IR spectrometer through CPE scheme.

R E F E R E N C E S

[1] Yamanaka S, Brindley GW. Hydroxy-nickel interlayering in montmorillonite by titration method. Clays Clay Miner 1978;26:21-4.

[2] Ohtsuka K, Suda M, Tsunoda M, Ono M. Synthesis of metal hydroxide-layer silicate intercalation compounds (metal $=\mathrm{Mg}$ (II), $\mathrm{Ca}$ (II), $\mathrm{Mn}$ (II), $\mathrm{Fe}(\mathrm{II}), \mathrm{Co}(\mathrm{II}), \mathrm{Ni}(\mathrm{II}), \mathrm{Zn}$ (II), and Cd(II)). Chem Mater 1990;2:511-7.

[3] Yamanaka S, Senda K. Thermal evolution of nickel chloritelike phase derived from montmorillonite. Clay Sci 1993;9:99-107.

[4] Golub AS, Payen C, Protzenko GA, Novikov YN, Danot M. Nanocomposite materials consisting of alternating layers of molybdenum disulfide and cobalt or nickel hydroxides: magnetic characterization. Solid State Commun 1997;102:419-23.

[5] Yang X, Makita Y, Liu Z-H, Ooi K. Novel synthesis of layered graphite oxide-birnessite manganese oxide nanocomposite. Chem Mater 2003;15:1228-31.

[6] Xu Y, Feng Q Kajiyoshi K, Yanagisawa K. Hydrothermal intercalation reaction of nickel hydroxide into layered manganese oxide. Chem Mater 2002;14:697-703.

[7] Feng Q Honbu C, Yanagusawa K, Yamasaki N. Hydrothermal soft chemical reaction for formation of sandwich layered manganese oxide. Chem Mater 1999;11:2444-50.

[8] Feng Q Honbu C, Yanagusawa K, Yamasaki N, Komarneni S. Synthesis of $\mathrm{LiAl}_{2}(\mathrm{OH})_{6}{ }^{+}$intercalated montmorillonite by a 
hydrothermal soft chemical reaction. J Mater Chem 2000;10:483-8.

[9] Németh J, Dékány I, Süvegh K, Marek T, Klencsár Z, Vértes A, et al. Preparation and structural properties of tin oxidemontmorillonite nanocomposites. Langmuir 2003;19:3762-9.

[10] Xie J, Zhang Q, Shi P, Liu Q. Electrochemical properties and structure of $\mathrm{Ni}(\mathrm{OH})_{2}$-graphite intercalation compounds. Solid State Ionics 1999;121:67-71.

[11] Nethravathi C, Ravishankar N, Shivakumara C, Rajamathi M. Nanocomposites of $\alpha$-hydroxides of nickel and cobalt by delamination and co-stacking: enhanced stability of $\alpha$-motifs in alkaline medium and electrochemical behaviour. J Power Sources 2007;172:970-4.

[12] Venugopal BR, Shivakumara C, Rajamathi M. A composite of layered double hydroxides obtained through random costacking of layers from $\mathrm{Mg}-\mathrm{Al}$ and Co-Al-LDHs by delamination-restacking: thermal decomposition and reconstruction behaviour. Solid State Sci 2007;9:287-94.

[13] Venugopal BR, Ravishankar N, Rajamathi M. Interstratification of trioctahedral and dioctahedral smectites through delamination and costacking. J Colloid Interface Sci 2008;324:80-5.

[14] Nethravathi C, Viswanath B, Ravishankar N, Rajamathi M. The production of smectite clay/graphene composites through delamination and co-stacking. Carbon 2008;46:1773-81.

[15] Hornok V, Erdohelyi A, Dekany I. Preparation of ultrathin membranes by layer-by-layer (LBL) deposition of oppositely charged inorganic colloids. Colloid Polym Sci 2006;284: 611-9.

[16] Li L, Ma R, Ebina Y, Fukuda K, Takada K, Sasaki T. Layer-bylayer assembly and spontaneous flocculation of oppositely charged oxide and hydroxide nanosheets into inorganic sandwich layered materials. J Am Chem Soc 2007;129:8000-7.

[17] Nethravathi C, Rajamathi JT, Ravishankar N, Shivakumara C, Rajamathi M. Graphite oxide-intercalated anionic clay and its decomposition to inorganic material nanocomposites. Langmuir 2008;24:8240-4.

[18] Geim AK, Novoselov KS. The rise of graphene. Nat. Mater 2007;6:183-91.

[19] Stankovich S, Dikin DA, Dommett GHB, Kohlhaas KM, Zimmey EJ, Stach EA, et al. Graphene-based composite materials. Nature 2006;442:282-6.

[20] Cassagneau T, Fendler JH. Preparation and layer-by-layer selfassembly of silver nanoparticles capped by graphite oxide nanosheets. J Phys Chem B 1999;103:1789-93.

[21] Cassagneau T, Fendler JH. High density rechargeable lithiumion batteries self-assembled from graphite oxide nanoplatelets and polyelectrolytes. Adv Mater 1998;10:877-81.

[22] Frackowiak E, Beguin F. Carbon materials for the electrochemical storage of energy in capacitors. Carbon 2001;39:937-50.

[23] Croft RC. Lamellar compounds of graphite. Quart Rev 1960;14(1):11-3.

[24] Nethravathi C, Rajamathi M. Delamination, colloidal dispersion and reassembly of alkylamine intercalated graphite oxide in alcohols. Carbon 2006;44:2635-41.
[25] Dékány I, Krüger-Grasser R, Weiss A. Selective liquid sorption properties of hydrophobized graphite oxide nanostructures. Colloid Polym Sci 1998;276:570-6.

[26] Matsuo Y, Niwa T, Sugie Y. Preparation and characterisation of cationic surfactant-intercalated graphite oxide. Carbon 1999;37:897-901.

[27] Falk SU, Salkind AJ. Alkaline storage batteries. New York: Wiley; 1969.

[28] Oliva P, Leonardi J, Laurent JF, Delmas C, Braconnier JJ, Figlarz $\mathrm{M}$, et al. Review of the structure and the electrochemistry of nickel hydroxides and oxyhydroxides. J Power Sources 1982;8:229-55.

[29] Delmas C, Faure C, Gautier L, Demourgues-Guerlou L, Rougier A. The nickel hydroxide electrode from the solid-state chemistry point of view. Philos Trans R Soc Lond A 1996;354:1545-54.

[30] Kamath PV, Therese GH, Gopalakrishnan AJ. On the existence of hydrotalcite-like phases in the absence of trivalent cations. J Solid State Chem 1997;128:38-41.

[31] Nethravathi C, Harichandran G, Shivakumara C, Ravishankar $\mathrm{N}$, Rajamathi M. Surfactant intercalated $\alpha$-hydroxides of cobalt and nickel and their delamination-restacking behaviour in organic media. J Colloid Interface Sci 2005;288:629-33.

[32] Hummers WS, Offeman RE. Preparation of graphite oxide. J Am Chem Soc 1958;80:1339.

[33] Liu ZH, Wang Z-M, Yang X, Ooi K. Intercalation of organic ammonium ions into layered graphite oxide. Langmuir 2002;18:4926-32.

[34] Warren BE, Bodenstein P. The diffraction pattern of fine particle carbon blacks. Acta Crystallogr 1965;18:282-6.

[35] Rajamathi M, Kamath PV, Seshadri R. Polymorphism in nickel hydroxide: role of interstratification. J Mater Chem 2000;10:503-6.

[36] Herrera-Alonso M, Abdala AA, McAllister MJ, Aksay IA, Prud'homme RK. Intercalation and stitching of graphite oxide with diaminoalkanes. Langmuir 2007;23:10644-9.

[37] Matsuo Y, Nishino Y, Fukutsuka T, Sugie Y. Introduction of amino groups into the interlayer space of graphite oxide using 3-aminopropylethoxysilanes. Carbon 2007;45:1384-90.

[38] Szabo T, Tombacz E, Illes E, Dekany I. Enhanced acidity and $\mathrm{pH}$-dependent surface charge characterisation of successively oxidized graphite oxides. Carbon 2006;44:537-45.

[39] Snyder RG, Strauss HL, Elliger CA. Carbon-hydrogen stretching modes and the structure of n-alkyl chains 1. Long, disordered chains. J Phys Chem 1982;86:5145-50.

[40] MacPhail RA, Strauss HL, Snyder RG, Elliger CA. Carbonhydrogen stretching modes and the structure of n-alkyl chains 2. Long, all-trans chains. J Phys Chem 1984;88:334-41.

[41] Matsuo Y, Sugie Y. Preparation, structure and electrochemical property of pyrolytic carbon from graphite oxide. Carbon 1998;36:301-3.

[42] Nethravathi C, Sen S, Ravishankar N, Rajamathi M, Pietzonka C, Harbrecht B. Ferrimagnetic nanogranular $\mathrm{Co}_{3} \mathrm{O}_{4}$ through solvothermal decomposition of colloidally dispersed monolayers of $\alpha$-cobalt hydroxide. J Phys Chem B 2005;109:11468-72. 